\title{
Fiber optical dose rate measurement based on the luminescence of beryllium oxide
}

\author{
Tobias Teichmann, Jan Sponner and Jürgen Henniger, TU Dresden
}

\begin{abstract}
This work presents a fiber optical dose rate measurement system based on the radioluminescence and optically stimulated luminescence of beryllium oxide. The system consists of a small, radiation sensitive probe which is coupled to a light detection unit with a long and flexible light guide. Exposing the beryllium oxide probe to ionizing radiation results in the emission of light with an intensity which is proportional to the dose rate. Additionally, optically stimulated luminescence can be used to obtain dose and dose rate information during irradiation or retrospectively. The system is capable of real time dose rate measurements in fields of high dose rates and dose rate gradients and in complex, narrow geometries. This enables the application for radiation protection measurements as well as for quality control in radiotherapy. One inherent drawback of fiber optical dosimetry systems is the generation of Cherenkov radiation and luminescence in the light guide itself when it is exposed to ionizing radiation. This so called "stem" effect leads to an additional signal which introduces a deviation in the dose rate measurement and reduces the spatial resolution of the system, hence it has to be removed. The current system uses temporal discrimination of the effect for radioluminescence measurements in pulsed radiation fields and modulated optically stimulated luminescence for continuous irradiation conditions. This work gives an overview of the major results and discusses new-found obstacles of the applied methods of stem discrimination.
\end{abstract}

$\begin{array}{crrr}\text { Index Terms-Beryllium } & \text { oxide, Fiber optic dose rate } \\ \text { measurements, } & \text { Optically } & \text { Stimulated } & \text { Luminescence, }\end{array}$ Radioluminescence

\section{INTRODUCTION}

$\mathrm{F}$ IBER Optical Dosimeters (FODs) allow real time dose rate measurements with high spatial resolution. FODs consist of a small, radiation sensitive probe which is coupled to a light detection unit with a long and flexible light guide. Exposing the probe to ionizing radiation results in the emission of radioluminescence light (RL) with a characteristic spectrum and an intensity which is, under certain circumstances, proportional to the dose rate. Additional dose

ANIMMA 2017 Conference Proceedings, submitted June 27, 2017.

T. Teichmann is with the Radiation Physics Group at the Institute of Nuclear and Particle Physics, TU Dresden, Zellescher Weg 19, 01069, Dresden, Germany (e-mail: teichmann@asp.tu-dresden.de).

J. Sponner was with the Radiation Physics Group at the Institute of Nuclear and Particle Physics, TU Dresden, Zellescher Weg 19, 01069, Dresden, Germany (e-mail: sponner@asp.tu-dresden.de).

J. Henniger is with the Radiation Physics Group at the Institute of Nuclear and Particle Physics, TU Dresden, Zellescher Weg 19, 01069, Dresden, Germany (e-mail: henniger@asp.tu-dresden.de). and dose rate information can be obtained by in-beam or retrospective optical stimulation of the probe with blue light which leads to optically stimulated luminescence (OSL).

Due to the separation of radiation sensitive volume and light detection and stimulation unit, the system qualifies for measurements in fields of high dose rates and dose rate gradients and in complex and narrow geometries without exposing the operator or the susceptible electronics. Possible applications are radiation protection measurements in facilities for the generation of ionizing radiation and the surveillance and dismantling of nuclear facilities as well as quality control in radiotherapy and nuclear medicine.

One inherent drawback of fiber optical dosimetry systems is the generation of Cherenkov radiation (CR) and luminescence in the light guide itself when it is exposed to ionizing radiation. This "stem" effect results in an additional signal component which is also detected by the PMT. This causes a deviation of the dose rate measurement and reduces the spatial resolution of the system. Therefore the stem effect must be removed. Promising solutions to do so have been investigated in the past [1-3].

In the current system, based on beryllium oxide, two different methods of temporal discrimination of the effect for RL measurements in pulsed radiation fields (already introduced in [4]) have been tested. Furthermore, a combination of optical filtering and modulated OSL, which is an enhancement of [5], has been applied to measurements under continuous irradiation conditions. This work gives an overview of the major results and discusses new-found obstacles of the applied methods of stem discrimination in pulsed radiation fields.

\section{Material AND Methods}

\section{A. Dosimetry System}

The presented dosimetry system uses beryllium oxide $(\mathrm{BeO})$ in form of a sintered ceramic cylinder (height $1 \mathrm{~mm}$, diameter $1 \mathrm{~mm}$, Thermalox $® 995$, Materion) as a radiation sensitive probe. The well-known OSL phosphor has been investigated and applied for integrating dose measurements for decades. In the recent past $\mathrm{BeO}$ gained greater attention in the field of FODs [4-8]. The material is almost tissue equivalent with an effective atomic number of 7.2. Its luminescence emission spectrum has a broad global maximum at $370 \mathrm{~nm}$. The luminescence life time is $27 \mu \mathrm{s}$ at room temperature [9].

The $\mathrm{BeO}$ detector is coupled to the tip of a PMMA light guide (diameter $1 \mathrm{~mm}$, length $15 \mathrm{~m}$ ), which is shielded from 
ambient light with a black coating. The RL and OSL, generated in the $\mathrm{BeO}$ during irradiation and/or optical stimulation, passes the light guide and is detected with a PMT (H10682-210, Hamamatsu) operated in single photon counting mode. In contrast to the previous measurements in [4], an adjustable mechanical aperture was added to reduce the intensity of the luminescence light from the probe in order to measure the influence of the stem signal in the linear detection range of the PMT.

The logical pulses originating the light detector are sampled with $100 \mathrm{MHz}$ by an FPGA. Each incoming pulse is assigned with a time stamp, representing its arrival time since the start of the measurement.

To perform additional OSL measurements, stimulation light from a diode laser (peak wavelength $450 \mathrm{~nm}$, Osram) is coupled into the light guide using a $400 \mathrm{~nm}$ dichroic beam splitter (Edmund Optics) and a fiber collimator (Hellma Analytics). The stimulation current can be modulated externally. The laser diode features temperature stabilization. Its maximum optical output power is $1.6 \mathrm{~W}$. However, absorption of stimulation light in the light guide and fiber connector leads to a raise in temperature, which can affect the transmission of the components. In order to prevent the resulting fading effects in the responsivity of the probes, the power is limited to $600 \mathrm{~mW}$ in the present measurements.

\section{B. Methods of Gated Discrimination}

In contrast to the luminescence life time of $\mathrm{BeO}(27 \mu \mathrm{s})$ the decay time of the stem signal is much smaller, hence the RL signal of $\mathrm{BeO}$ is still present shortly after irradiation, when the stem component is already negligible [4]. For measurements in pulsed radiation fields, this is exploited to generate a dose rate proportional signal, which is independent of the stem effect.

Two methods, i.e. "trigger and discriminator" and "artificial dead time", have been investigated to distinguish irradiation pulses and the time intervals between pulses (the latter providing the dose rate information) on the basis of the difference in event rates.

The first approach uses the distance between sequential time stamps to identify the irradiation pulses. The system stays triggered as long as the time stamp distances are shorter than a preset threshold $\Delta_{\mathrm{th}}$. After the end of the irradiation pulse and a following discrimination time $T_{\mathrm{d}}$, the stem component of the signal has decayed and the measured signal is only RL. The procedure is illustrated in Fig. 1.

The second method generates histograms of time stamp distances. Short distances are more likely to occur during an irradiation pulse, while longer distances are assigned to the intervals between irradiation. Introducing an artificial dead time $\Delta_{\mathrm{d}}$ and neglecting all smaller time stamp distances will result in a signal, which is associated with the part between irradiation pulses. Examples of time stamp distance histograms are displayed in Fig. 4.

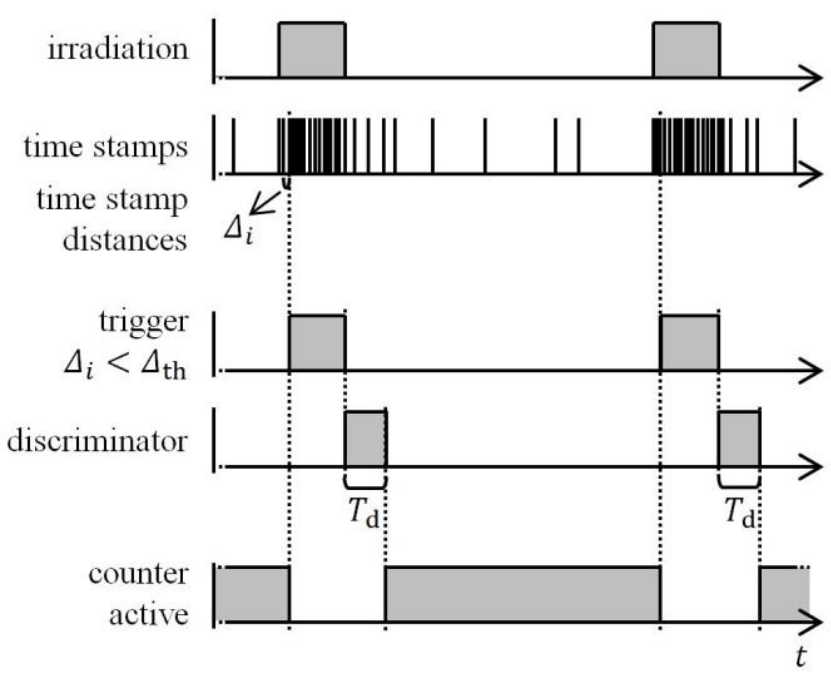

Fig. 1. Trigger and discriminator for gated measurement of the RL signal

\section{Irradiation}

Pulsed irradiation has been performed in a $30 \mathrm{~cm} \times 30 \mathrm{~cm} \times 20 \mathrm{~cm}$ solid water phantom with a $6 \mathrm{MV}$ medical linear accelerator (Elekta). The maximum nominal dose rate in the isocenter was $550 \mathrm{cGy} / \mathrm{min}$. A second pulsed $150 \mathrm{keV}$ X-ray source (Golden Engineering) with $30 \mu \mathrm{Gy}$ per pulse in $30 \mathrm{~cm}$ distance, a pulse length of $50 \mathrm{~ns}$, and a repetition rate of $25 \mathrm{~Hz}$ was also applied.

\section{RESUlts AND Discussion}

Using the two methods of gated discrimination, profile measurements in the pulsed radiation field of the linac as well as relative depth dose curve measurements in the field of the pulsed X-ray source have been performed. The motivation is to gain further information about the influence of the parameters of both methods on the output of the system at different irradiation conditions.

For the profile measurements the probe was pushed through a $20 \mathrm{~cm} \times 20 \mathrm{~cm}$ irradiation field, increasing the length of irradiated light guide with each position. The depth in the phantom was $10 \mathrm{~cm}$ for all positions.

Depth dose curves were measured by varying the position of the adapter plate of the probe within the stack of solid water slabs. Another dose depth curve has been measured with a MiniDos OSL dosimeter [10] as a reference.

\section{A. Trigger and Discriminator}

The influence of the discrimination time on the profile measurement and the relative dose depth curve is presented in Fig. 2 and Fig. 3. 


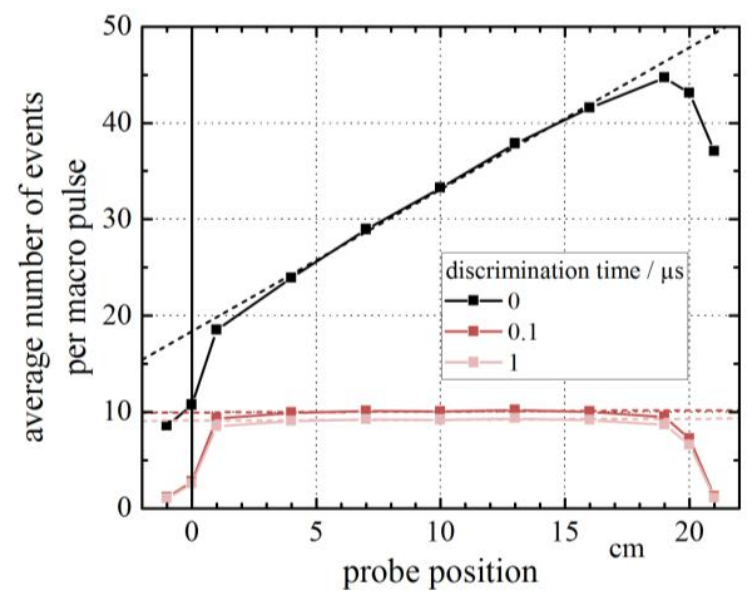

Fig. 2. Measurement of a dose profile in a $20 \mathrm{~cm} \times 20 \mathrm{~cm}$ irradiation field of a medical linac. Influence of the discrimination time. Mean of $\sim 15000$ macro pulses each.

With increasing probe position, i.e. increasing length of irradiated light guide, the unfiltered signal (discrimination time $=0$ ) rises due to the stem effect. The introduction of a dead time of 100 ns leads to a signal with no detectable stem influence left. Further extension of the discrimination time only results in a reduced signal but has no benefit to the stem suppression.

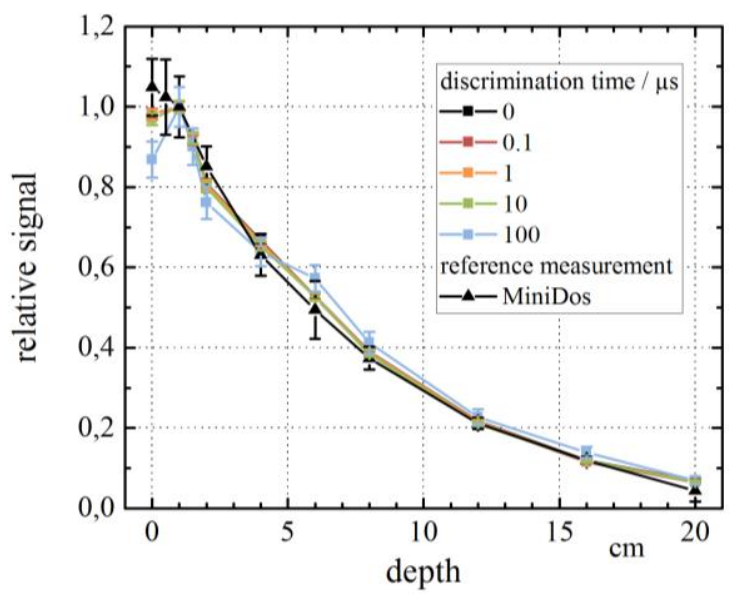

Fig. 3. Measurement of the depth dose curve with the trigger discriminator method in the field of a pulsed X-ray source. Reference measurement with integrating MiniDos OSL dosimeters. Mean of 200 Pulses each.

The dose depth curves are in agreement with the reference for discrimination times up to $10 \mu \mathrm{s}$. Larger values result in deviations due to statistical fluctuation because of the small signal. There is a difference between RL probe and reference for the entrance dose at depth 0 . The RL probe detects a small build-up effect whereas the reference shows a strictly decreasing behavior. This is due to the different construction of both detectors. The encapsulation of the RL probe is thinner than the one of the reference. So for the RL probe the conditions for secondary particle equilibrium are not met at the surface of the phantom.

To detect all irradiation pulses of the linac, the trigger threshold had to be adjusted with increasing depth. This is caused by the influence of the absorbed dose per irradiation pulse on the measured event rate during the pulse. The problem can be solved in several ways. To obtain a reliable, independent trigger, it is possible to increase the length of irradiated light guide on purpose and use the stem signal for triggering as in [4] or install a second reference fiber with independent light detection which is sensitive on the scattered radiation in the treatment room. A third possibility would be the use of an external trigger signal provided by the linac.

\section{B. Artificial Dead Timer}

The same profile measurements as described previously have been analyzed in terms of time stamp distances. Fig. 4 displays the corresponding histograms.

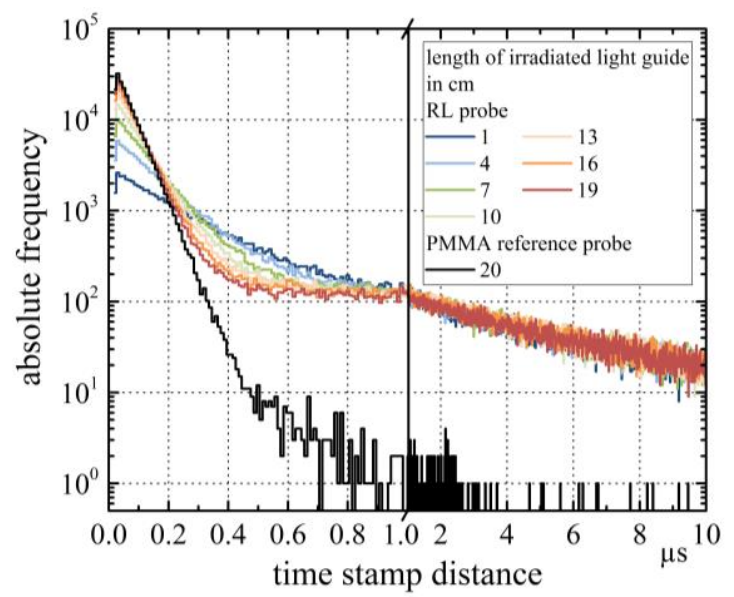

Fig. 4. Histograms of time stamp distances for $\mathrm{BeO}$ and PMMA reference probe. Dependency on the length of irradiated light guide. Pulsed irradiation with $550 \mathrm{cGy} / \mathrm{min}$ to a total absorbed dose of $2 \mathrm{~Gy}$, each measurement.

The distribution of time stamp distances below $1 \mu \mathrm{s}$ changes with varying length of irradiated light guide, whereas the distribution for larger distances remains unaffected. An additional measurement with a PMMA reference probe without $\mathrm{BeO}$ detector represents the stem only signal whose main contribution is at distances below $1 \mu \mathrm{s}$.

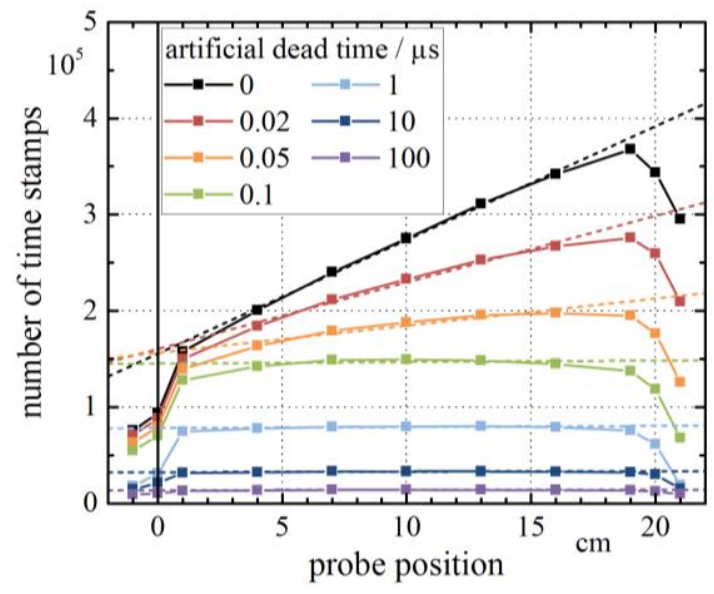

Fig. 5. Influence of the artificial dead time on the measurement of a dose profile in $20 \mathrm{~cm} \times 20 \mathrm{~cm}$ irradiation field of a medical linac. 
Applying the method of artificial dead time, the profiles, displayed in Fig. 5 can be obtained. Without artificial dead time the full histograms contribute to the signal and the stem influence is clearly visible. Increasing the dead time leads to a reduction of the effect, but also to a smaller total signal.

The quality of stem discrimination can be quantized, by calculating the ratio between the generated stem per $\mathrm{cm}$ irradiated light guide (which is represented by the slope of the profiles in Fig. 5) and the pure RL signal (which is given by the value of the linear approximation at position $0 \mathrm{~cm}$ ). Since the real beam profile has no perfect rectangular shape, only the central part $(4-16 \mathrm{~cm})$ has been used for the linear approximation. A stem to $\mathrm{RL}$ ratio of $1 \% / \mathrm{cm}$ means that, irradiated at the same dose rate, $100 \mathrm{~cm}$ of light guide generate the same event rate as the $\mathrm{RL}$ originating from the $\mathrm{BeO}$ detector at the tip of the probe. The efficiency of the stem discrimination in dependency of the artificial dead time is shown in Fig. 6. The best quality of stem discrimination is achieved with $100 \mathrm{~ns}$ artificial dead time. The minimum stem to $\mathrm{RL}$ ratio is $0.4 \% / \mathrm{cm}$.

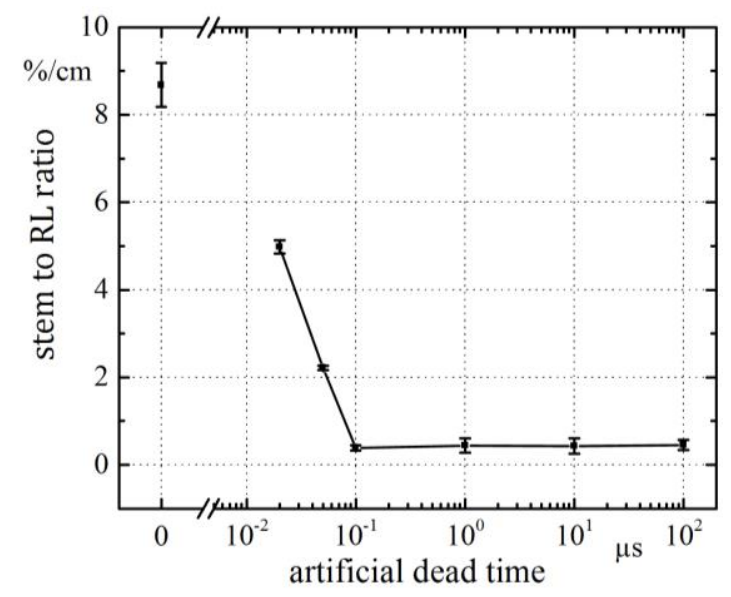

Fig. 6. Efficiency of the stem discrimination in dependency of the artificial dead time.

The results of the depth dose curve measurements with artificial dead time are displayed in Fig. 7. For dead times longer than $0.1 \mu \mathrm{s}$ there is a strong deviation from the reference. Also the same build-up effect as in Fig. 3 is visible.

For dead times of $0.1 \mu \mathrm{s}$ and smaller the depth dose curve follow the reference in rough accordance. But there is a noticeable overestimation of the dose curve for larger depths. The explanation is the following. The shape of the histogram is dependent on the dose per pulse. A smaller dose per irradiation pulse results in a smaller event rate during irradiation pulses which causes a right shift of the histogram. With a constant preset value for the artificial dead time, more time stamp distances contribute to the signal. This problem has to be investigated next. A possible solution would be a variable artificial dead time which is adjusted, based on an estimation of the event rate during irradiation pulses.



Fig. 7. Depth dose curve measurement with artificial dead time in the field of a pulsed X-ray source. Measurement with integrating MiniDos dosimeters as reference.

\section{CONCLUSION}

Both methods "trigger and discriminator" and "artificial dead time" successfully discriminate the stem effect, which is the main obstacle in fiber optic dose rate measurements. The influence of the parameters, trigger threshold, discrimination time and artificial dead time, has been investigated, leaving open the dependency of the histograms on the dose per pulse.

\section{ACKNOWLEDGMENT}

The authors thank H. Blank and the practice for radiotherapy and radiooncology Distler in Pirna, Germany for the possibility to perform measurements at their medical linear accelerator.

\section{REFERENCES}

[1] S. F. de Boer, A. S. Beddar, and J. A. Rawlinson, "Optical filtering and spectral measurements of radiation-induced light in plastic scintillation dosimetry“, Phys. Med. Biol., vol. 38, no. 7, pp. 945-958, Apr. 1993.

[2] J. C. Polf, et al., "Real-time luminescence from $\mathrm{A} 12 \mathrm{O} 3$ fiber dosimeters“, Radiat. Meas., vol. 38, no. 2, pp. 227-240, Apr. 2004.

[3] R. Gaza et al., "A fiber-dosimetry method based on OSL from Al2O3:C for radiotherapy applications", Radiat. Meas., vol. 38, no. 46, pp. 809-812, Aug. 2004.

[4] T. Teichmann et al., "Gated discrimination of the stem signal in pulsed radiation fields for a fiber optic dosimetry system based on the radioluminescence of beryllium oxide", Radiat. Meas., to be published. DOI: $10.1016 /$ j.radmeas.2017.03.046.

[5] T. Teichmann et al., "Real time dose rate measurements with fiber optic probes based on the RL and OSL of beryllium oxide", Radiat. Meas., vol. 90, pp. 201-204, July 2016.

[6] A. M. C. Santos, et al., "Characterization of a real-time fibre-coupled beryllium oxide (BeO) luminescence dosimeter in X-ray beams", Radiat. Meas., vol. 53-54, pp. 1-7, June 2013.

[7] A. M. C. Santos, M. Mohammadi, and S. Afshar V., "Investigation of a fibre-coupled beryllium oxide $(\mathrm{BeO})$ ceramic luminescence dosimetry system", Radiat. Meas., vol. 70, pp. 52-58, Nov. 2014.

[8] A. M. C. Santos, M. Mohammadi, and S. Afshar V., "Evaluation of a real-time $\mathrm{BeO}$ ceramic fiber-coupled luminescence dosimetry system for dose verification of high dose rate brachytherapy”, Med. Phys., vol. 42, no. 11, pp. 6349-6356, Oct. 2015.

[9] E. G. Yukihara, "Luminescence properties of $\mathrm{BeO}$ optically stimulated luminescence (OSL) detectors“, Radiat. Meas., vol. 46, no. 6-7, pp. 580-587, June 2011.

[10] A. Jahn et al., "The BeOmax system - Dosimetry using OSL of BeO for several applications", Radiat. Meas., vol. 56, pp. 324-327, Sep. 2013. 